\title{
Stereotactic body radiotherapy (SBRT) for adrenal metastases of oligometastatic or oligoprogressive tumor patients
}

Laila König $^{1,2,3^{*}}$ (D), Matthias F. Häfner ${ }^{1,2,3}$, Sonja Katayama ${ }^{1,2,3}$, Stefan A. Koerber ${ }^{1,2,3}$, Eric Tonndorf-Martini ${ }^{1,2,3,4}$, Denise Bernhardt ${ }^{1,2,3}$, Bastian von Nettelbladt ${ }^{1,2,3}$, Fabian Weykamp ${ }^{1,2,3}$, Philipp Hoegen ${ }^{1,2,3}$, Sebastian Klüter ${ }^{1,2,3}$, Matthew S. Susko ${ }^{7}$, Jürgen Debus ${ }^{1,2,3,4,5,6}$ and Juliane Hörner-Rieber ${ }^{1,2,3,4}$

\begin{abstract}
Introduction: Local ablative treatment strategies are frequently offered to patients diagnosed with oligometastatic disease. Stereotactic body radiotherapy (SBRT), as ablative treatment option, is well established for lung and liver metastases, whereas for isolated adrenal gland metastases the level of evidence is scarce.

Material and methods: This single-institution analysis of oligometastatic or oligoprogressive disease was limited to patients who received SBRT to adrenal metastasis between 2012 and 2019. Patient, tumor, treatment characteristics, and dosimetric parameters were analyzed for evaluation of their effect on survival outcomes.

Results: During the period of review 28 patients received ablative SBRT to their adrenal gland metastases. Most common primary tumors were non-small cell lung cancers (46\%) with most patients diagnosed with a single adrenal gland metastasis (61\%), which occurred after a median time of 14 months. SBRT was delivered to a median biological effective dose at $\alpha / \beta$ of $10\left(B_{10}\right)$ of $75 \mathrm{~Gy}$ (range: 58-151 Gy). Median gross tumor volume (GTV) and median planning target volume (PTV) were 42 and $111 \mathrm{~mL}$, respectively. The homogeneity and conformity indices were 1.17 (range: 1.04-1.64) and 0.5 (range: 0.4 .0 .99 ), respectively, with the conformity index being affected by dose restrictions to organs at risk (OARs) in $50 \%$ of the patients. Overall response rate based on RECIST criteria was $86 \%$ $(C R=29 \%, P R=57 \%)$ with 2-year local control $(L C)$ of $84.8 \%$, 2-year progression-free survival (PFS) of 26.3\%, and 1and 2-year overall survival (OS) of 46.6 and $32.0 \%$, respectively. During follow up, only two local recurrences occurred. A trend for superior LC was seen if $\mathrm{BED}_{10}$ was $\geq 75 \mathrm{~Gy}(p=0.101)$ or if the PTV was $<100 \mathrm{ml}(p=0.072)$. SBRT was tolerated well with only mild toxicity.

Conclusion: SBRT for adrenal metastases resulted in promising LC with low toxicity. Treatment response appeared to be superior, if SBRT was applied with higher BED. As the close proximity of OARs often limits the application of sufficiently high doses, further dose escalations strategies and techniques should be investigated in future.
\end{abstract}

Keywords: Adrenal gland metastases, Stereotactic body radiotherapy (SBRT), Image-guided radiotherapy, Oligometastases, Oligoprogression

\footnotetext{
* Correspondence: laila.koenig@med.uni-heidelberg.de

${ }^{1}$ Department of Radiation Oncology, Heidelberg University Hospital, Im Neuenheimer Feld 400, 69120 Heidelberg, Germany

${ }^{2}$ Heidelberg Institute of Radiation Oncology (HIRO), Heidelberg, Germany

Full list of author information is available at the end of the article
}

(c) The Author(s). 2020 Open Access This article is distributed under the terms of the Creative Commons Attribution 4.0 International License (http://creativecommons.org/licenses/by/4.0/), which permits unrestricted use, distribution, and reproduction in any medium, provided you give appropriate credit to the original author(s) and the source, provide a link to the Creative Commons license, and indicate if changes were made. The Creative Commons Public Domain Dedication waiver (http://creativecommons.org/publicdomain/zero/1.0/) applies to the data made available in this article, unless otherwise stated. 


\section{Introduction}

The adrenal glands represent a common site for cancer metastases for a variety of primary tumors, with historic autopsy series reporting an incidence of 9-27\% [1]. The majority of adrenal metastases are accounted for by primary lung tumors, however breast, kidney, stomach, hepatobiliary carcinoma, and melanoma have also been found to metastasize to the adrenal glands [2, 3]. Traditionally, local therapies like surgical resection or radiotherapy were reserved for palliation of symptomatic lesions, with external beam radiotherapy demonstrating good response rates and high rates of pain relief (40-80\%) [4-6].

In the contemporary setting, local ablative treatment strategies for metastases are frequently offered for patients diagnosed in an oligometastatic or oligoprogressive tumors, irrespective of symptomatology [7-9]. Two recently published prospective phase II studies demonstrate that the addition of local ablative treatment to standard of care palliative therapy for oligometastatic tumor patients not only prolonged progression-free survival (PFS), but also overall survival (OS) [10, 11]. Additionally, some surgical series report superior outcomes following adrenalectomy in selected patients with isolated adrenal gland metastases [12-15]. However, many patients with adrenal gland metastases are considered medically inoperable due to severe comorbidities or the location or the extent of their metastases [16]. For these patients, stereotactic body radiotherapy (SBRT) offers an excellent, noninvasive treatment alternative $[4,16]$.

In contrast to conventionally fractionated radiotherapy, SBRT allows for the delivery of highly conformal doses or radiation, enabling tumor ablation. While SBRT is well established and widely applied in the treatment of pulmonary and hepatic oligometastases [17-20], the literature for adrenal metastases is scarce. Predominantly small and retrospective studies exist which do not primarily focus on oligometastatic or oligoprogressive disease, but also include patients treated with palliative intent [21-27]. Furthermore, these studies only report limited details on radiotherapy planning and requirement for forced dose restrictions due to the close proximity to radiosensitive organs-at risks (OARs). The aim of the current study was therefore to evaluate outcomes and potentially related dosimetric parameters in oligometastatic or oligoprogressive patients treated with SBRT for adrenal metastases.

\section{Materials and methods}

\section{Patient population}

This study is a retrospective, single-institution analysis of oligometastatic (1-5 metastases), or oligoprogressive patients, who were treated for adrenal metastases with SBRT between October 2012 and January 2019. Institutional databases were reviewed for demographic, pathologic, radiologic, and treatment-related information. Ethical approval was sought from the Ethical Committee of the University Hospital of Heidelberg (S-627/2019). Patients were excluded from the analysis if they were not treated with SBRT but with palliative intent or palliative doses. SBRT was defined as an ablative dose with single fraction doses $>4$ Gy and number of fractions < 10. All patients were either classified medically or technically inoperable. No patient suffered from symptoms related to the adrenal gland metastasis.

\section{Planning and treatment features}

For treatment planning, computed tomography (CT) scans with and without contrast and a slice thickness of $3 \mathrm{~mm}$ were acquired under shallow breathing. To account for respiratory motion the preference was to obtain a 4dimensional (4-D) CT scan during normal breathing whenever feasible $(n=24$ patients with $4 \mathrm{D}-\mathrm{CT})$. Contrastenhanced abdominal magnetic tomography imaging (MRI) and fluoro-deoxy-glucose positron emission tomography (FDG-PET) imaging were further applied for treatment planning in 7 patients each. Gross tumor volumes (GTV) included the macroscopic tumor on the contrast-enhanced CT, FDG-PET-CT and MR scans, with an internal target volume (ITV) being defined based on the particular tumor motion assessed on the 4-D-CT scans. To account for microscopic infiltration, a $5 \mathrm{~mm}$ safety margin was applied to form the clinical target volume (CTV). An additional planning target volume (PTV) margin of $2 \mathrm{~mm}$ was further added to account for positioning insecurities. If no 4-D-CT data was available, a PTV margin of $10 \mathrm{~mm}$ was used. Total dose and fractionation schemes were at the discretion of the treating physician and dependent on the adjacent normal tissue tolerances. As adrenal SBRT performed with higher biological effective doses (BED) is associated with superior local control (LC) treatment with maximally safe doses was generally pursued [26]. Applied dose constraints were in line with published guidelines $[18,20,28]$ : e.g. liver dose to $\geq 700 \mathrm{ml}$ $<15$ Gy (3 fractions) and < 18.5 Gy (5 fraction) < 24 Gy (10 fractions), spinal cord $\mathrm{D}_{0.1 \mathrm{cc}}<21.5 \mathrm{~Gy}$ (3 fractions), <30Gy (5 fractions) <35 Gy (10 fractions), stomach/small bowel $\mathrm{D}_{0.5 \mathrm{cc}}<25.2 \mathrm{~Gy}$ (3 fractions), <35 Gy (5 fractions) and $<43.5$ Gy (10 fractions). Volumetric image guidance for patient positioning using either $\mathrm{kV}$ cone beam CT (CBCT) or MV CT was performed before each treatment fraction.

The biological effective dose (BED) was calculated for correlating irradiated doses with clinical results: an $\alpha / \beta$ ratio of $10 \mathrm{~Gy}$ was assumed for the adrenal metastases. BED was determined using the linear-quadratic model [29]:

$$
\text { BED }(\text { Gy })=\text { fractional dose } \times \text { number of fractions }\left(1+\frac{\text { fractional dose }}{\alpha / \beta}\right)
$$

Further quantitative and qualitative dose evaluations were conducted with dose parameters being extracted from dose volume histograms (DVH) to asses target coverage, homogeneity and conformity of the PTV. For 
homogeneity index (HI), a formula was used, where D5\% and $\mathrm{D} 95 \%$ are the minimum doses in 5 and $95 \%$ of the target volume, respectively. The ideal HI value is 1 and increases as the plan becomes less homogeneous [30].

$$
\text { Homogeneity index }\left(\mathrm{HI}_{\mathbf{5} / 95}\right)=\frac{\mathrm{D}_{\mathbf{5} \%}}{\mathbf{D}_{\mathbf{9 5} \%}}
$$

The conformity index $(\mathrm{CI})$ was calculated according to the RTOG guidelines [31] by division of the PTV covered by the 95\% isodose (reference isodose) and the target volume itself. A value close to 1 corresponds to ideal conformity.

$$
\text { Conformity index }(\mathrm{CI})=\frac{\text { PTV }_{\mathbf{9 5}}}{\text { PTV }}
$$

\section{Outcome evaluation}

All patients received follow-up visits at the Heidelberg University Hospital or the Thoraxklinik Heidelberg and underwent a clinical examination and a contrastenhanced CT scan 6-8 weeks after the completion of treatment. This was repeated thereafter at 3- to 6-months intervals depending on the primary tumor entity and the overall performance status. Treatment-related toxicity was evaluated and classified according to CTCAE v5.0. Local control (LC) was defined as no progressive disease of the treated metastasis, with treatment response being scored using the Response Evaluation Criteria in Solid Tumors (version 1.1). Treatment response to adrenal SBRT was classified as complete response (CR), partial response $(\mathrm{PR})$, stable disease (SD), or progressive disease (PD).

\section{Statistical analysis}

The statistical analysis aimed to evaluate the efficacy of SBRT for adrenal metastases and to identify prognostic factors associated with outcomes. Survival analyses for local control (LC), progression-free survival (PFS) as well as overall survival (OS) following SBRT were performed using the Kaplan-Meier method. Univariate Cox proportional hazard models were used to assess the potential influence of all patient, tumor, and treatment characteristics on LC, PFS and OS. Multivariate analysis was not attempted due small sample size and the exploratory nature and of this analysis. A $p$-value $\leq 0.05$ was considered statistically significant. All statistical analyses were performed using the software SPSS 24.0 (IBM Corporation, Armonk, NY, USA).

\section{Results}

\section{Patient and treatment characteristics}

Between October 2012 and January 2019, 56 patients were treated with radiotherapy to their adrenal gland metastasis in our department. Twenty eight patients were excluded due to treatment with palliative doses. Of the remaining
28 patients available for further analysis, the median age was 63 years (range 48-91 years). Seventy-five percent of the patients met criteria of for an oligometastatic tumor stage with $0-5$ additional metastases. All additional metastases, as well as the primary tumor, received further treatment with surgery or radiotherapy with or without systemic therapy. Twenty-five percent of the patients were classified to have an oligoprogredient tumor state, meaning that all additional metastatic locations including the primary tumor had previously been controlled at the time of adrenal SBRT. Most common primary tumors were non-small cell lung cancers (46\%), small cell lung cancers (18\%) and renal cell carcinomas (7\%). The majority of patients were diagnosed with a single adrenal gland metastasis (61\%), which occurred metachronously after a median time of 14 months. Detailed patient and tumor characteristics are displayed in Table 1.

SBRT was delivered with a median dose of $50 \mathrm{~Gy}$ (range: 30-54) with a median number of fractions of 10 (range 312), corresponding to a median $\mathrm{BED}_{10}$ of $75 \mathrm{~Gy}$ (range 58-151 Gy). Doses were predominantly prescribed homogenously (93\%). Nevertheless, in $50 \%$ of the patients dose restrictions were necessary to spare surrounding OARs, such as the small bowel and the stomach.

\section{Planning and Dosimetric characteristics}

Median GTV and median PTV were $42 \mathrm{ml}$ (range 3-233 $\mathrm{ml}$ ) and $111 \mathrm{ml}$ (range 16-346 ml), respectively. Target volume coverage was analyzed using $D_{98}(\%)$ and $D_{2}(\%)$ of the prescribed doses to the GTV and PTV. Median $\mathrm{D}_{98}$ of the GTV and the PTV was 100\% (range: $70-120 \%$ ) and $80 \%$ (range: $60-100 \%$ ). Median $\mathrm{D}_{2}$ of the GTV and the PTV was found to be $100 \%$ (range: $100-130 \%$ ) and $100 \%$ (range: 100-140\%). To further objectify this analysis, we calculated homogeneity and conformity indices for all plans. The HI showed a median value of 1.17 (range: 1.04-1.64) and the CI a median of 0.5 (range 0.4.0.99), with the latter being effected by dose restrictions to OARs.

Delivery techniques comprised 3D conformal technique $(n=4)$, helical IMRT (intensity-modulated radiotherapy) $(n=13)$ and VMAT (volumetric modulated arc therapy) $(n=31)$. In 18 plans, flattening filter free (FFF) techniques were used.

\section{Treatment outcomes and toxicity}

Median follow up after initial tumor diagnosis was 36.4 months (range: 7.2-161.3 months) and 9.8 months (range 3.1-83.9 months) after adrenal SBRT. Clinical response after SBRT evaluated using RECIST criteria revealed a local overall response rate (ORR) of $86 \%(C R=29 \%, P R=$ $57 \%)$, illustrated in Table 2. Only two patients suffered from a local relapse, leading to a 1- and 2-year LC rate of $84.8 \%$. The first patient suffered from a NSCLC (adenocarcinoma) and his adrenal metastasis was treated with a 
Table 1 Patient, tumor and treatment characteristics

\begin{tabular}{|c|c|}
\hline & Total number of patients (\%) \\
\hline \multicolumn{2}{|l|}{ Gender } \\
\hline Male & $21(75.0 \%)$ \\
\hline Female & $7(25.0 \%)$ \\
\hline Median Age (range) in years & $63(48-91)$ \\
\hline Median KPS (range) in \% & $80(60-100 \%)$ \\
\hline \multicolumn{2}{|l|}{ Primary Tumor Site } \\
\hline NSCLC & $13(46.4 \%)$ \\
\hline $\mathrm{SCLC}$ & $5(17.9 \%)$ \\
\hline CRC & $1(3.6 \%)$ \\
\hline $\mathrm{HCC}$ & $1(3.6 \%)$ \\
\hline Breast cancer & $1(3.6 \%)$ \\
\hline Melanoma & $1(3.6 \%)$ \\
\hline RCC & $2(7.1 \%)$ \\
\hline Other & $4(14.3 \%)$ \\
\hline Oligometastatic & $21(75 \%)$ \\
\hline Oligoprogressive & $7(25 \%)$ \\
\hline \multicolumn{2}{|l|}{ Total no of metastatic sites } \\
\hline 0 & $17(60.7 \%)$ \\
\hline 1 & $2(7.1 \%)$ \\
\hline 2 & $2(7.1 \%)$ \\
\hline 3 & $1(3.6 \%)$ \\
\hline$>5$ & $6(21.4 \%)$ \\
\hline \multicolumn{2}{|l|}{ Laterality } \\
\hline Right & $14(50.0 \%)$ \\
\hline Left & $14(50.0 \%)$ \\
\hline \multicolumn{2}{|l|}{ Metastasis status } \\
\hline Synchronous & $10(35.7 \%)$ \\
\hline Metachronous & $18(64.3 \%)$ \\
\hline Median time from primary diagnosis to adrenal gland metastasis (range) in months & $14(0-102)$ \\
\hline \multicolumn{2}{|l|}{ Homogeneity distribution (\%) } \\
\hline Yes & $26(92.9 \%)$ \\
\hline No & $2(7.1 \%)$ \\
\hline \multicolumn{2}{|l|}{ Forced dose restriction (\%) } \\
\hline Yes & $14(50.0 \%)$ \\
\hline No & $14(50.0 \%)$ \\
\hline \multicolumn{2}{|l|}{ RT Technique } \\
\hline 3D conformal & $4(14.3 \%)$ \\
\hline Helical IMRT (Tomotherapy) & $13(46.4 \%)$ \\
\hline VMAT & $11(39.3 \%)$ \\
\hline \multicolumn{2}{|l|}{ FFF photons utilized } \\
\hline Yes & $18(64.3 \%)$ \\
\hline No & $10(35.7 \%)$ \\
\hline \multicolumn{2}{|l|}{ Concurrent systemic therapy } \\
\hline No & $19(67.9 \%)$ \\
\hline
\end{tabular}


Table 1 Patient, tumor and treatment characteristics (Continued)

\begin{tabular}{lll}
\hline & Total number of patients (\%) \\
\hline Yes & $9(32.1 \%)$ \\
$\quad$ Chemotherapy & $6(66.7 \%)$ & $2(22.2 \%)$ \\
$\quad$ Targeted therapy & $1(11.1 \%)$ & Median (range) \\
$\quad$ Immunotherapy & Mean & $50(30-54)$ \\
Total dose (Gy) & 47.3 & $10(3-12)$ \\
Fractions (n) & 9 & $5(4-18)$ \\
Single dose (Gy) & 5.6 & $75(57.6-151.2)$ \\
BED 10 (Gy) & 73.5 & $90(80-90)$ \\
Prescribed isodose line (\%) & 89 & 27 \\
Median GTV volume (range) in $\mathrm{cm}^{3}$ & 111 & $42(3-233)$ \\
Median PTV volume (range) in $\mathrm{cm}^{3}$ & $96(16-346)$ \\
\hline
\end{tabular}

KPS karnofsky performance score, NSCLC non-small cell lung cancer, SCLC small cell lung cancer, CRC colorectal cancer, HCC hepatocellular cancer, $R C C$ renal cell carcinoma, PTV planning target volume, $B E D_{10}$ biologically effective dose at $\alpha / \beta$ of $10, I M R T$ intensity-modulated radiotherapy, VMAT volumetric modulated arc therapy, FFF flattening filter free

BED of 67.5 Gy. He relapsed locally after 8.2 months without systemic failure and died due to pneumonia and pulmonary embolism some days after diagnosis of local failure. The second patient was also diagnosed with a NSCLC (adenocarcinoma), his adrenal metastasis was treated with a BED of $67.2 \mathrm{~Gy}$ and he relapsed only locally after 7 months. His progressive adrenal gland metastasis was resected (R1 status). However, 6 months after this operation, he was diagnosed with systemic failure (peritoneal carcinosis) and died another month after due to systemic progression.

The majority of patients (68\%) were diagnosed with distant relapse with a 1- and 2-year PFS rate of $26.3 \%$ (as shown on Fig. 1). During follow up, 18 patients died, resulting in a 1-and 2-year OS rate of 46.6 and $32.0 \%$. Most the deaths (83\%) were due to systemic tumor progression, while the other $17 \%$ were non-cancer-related. Median follow-up for patients who were still alive was higher with 26.1 months. Outcome data are displayed in Fig. 1. Various patient, tumor and treatment

Table 2 Treatment outcomes according to RECIST

\begin{tabular}{ll}
\hline & Total number of patients (\%) \\
\hline Clinical response & \\
PR & $8(28.6 \%)$ \\
SD & $16(57.1 \%)$ \\
PD & $2(7.1 \%)$ \\
Distant recurrence & $2(7.1 \%)$ \\
Yes & \\
No & $19(67.9 \%)$ \\
\hline
\end{tabular}

$C R$ complete remission, $P R$ partial remission, $S D$ stable disease, $P D$ progressive disease characteristics (age, sex, response to SBRT, BED, single dose, number of fractions, GTV and PTV, synchronous vs. metachronous disease, oligoprogredient vs. oligometastatic disease) were analyzed as prognostic factors for LC, PFS and OS. No significant prognostic correlations were observed. However, we detected a non-significant trend for superior LC if a BED $\geq 75 \mathrm{~Gy}(p=0.101)$ was applied or if PTV $<100 \mathrm{ml}(p=0.072)$ (see Fig. 1).

SBRT was tolerated well with only mild (grade I-II) acute toxicity in $32 \%$ of the patients. Most common acute side effects were $\mathrm{CTCAE}^{\circ} \mathrm{I}$ and II fatigue in 2 and 4 patients, respectively and $\mathrm{CTCAE}^{\circ}$ I and II gastrointestinal toxicity in 2 and one patients, which were nausea (one patient needed antiemetic medication $=$ grade 2 ) and loss of appetite without alteration in eating habits (grade 1). Late toxicity occurred in only 3 patients. No severe acute or chronic toxicity (>grade II) was observed during follow up. Two patients suffered from fatigue, only one patient was diagnosed with mild gastrointestinal toxicity, which was a loss of appetite without alteration in eating habits (grade 1). Detailed toxicity criteria are shown in Table 3.

\section{Discussion}

With the widespread use of regularly performed staging with CT or positron-emission-tomography (PET)-CT, and the growing recognition of the oligometastatic or oligoprogressive state in different tumor entities, the number of patients presenting with asymptomatic adrenal metastases has increased [32-34]. For these patients, surgical resection remains the gold standard, however, many patients are medically unfit for surgery, or their tumors are not technically resectable, and alternative local treatment approaches are required $[4,16,35]$. 


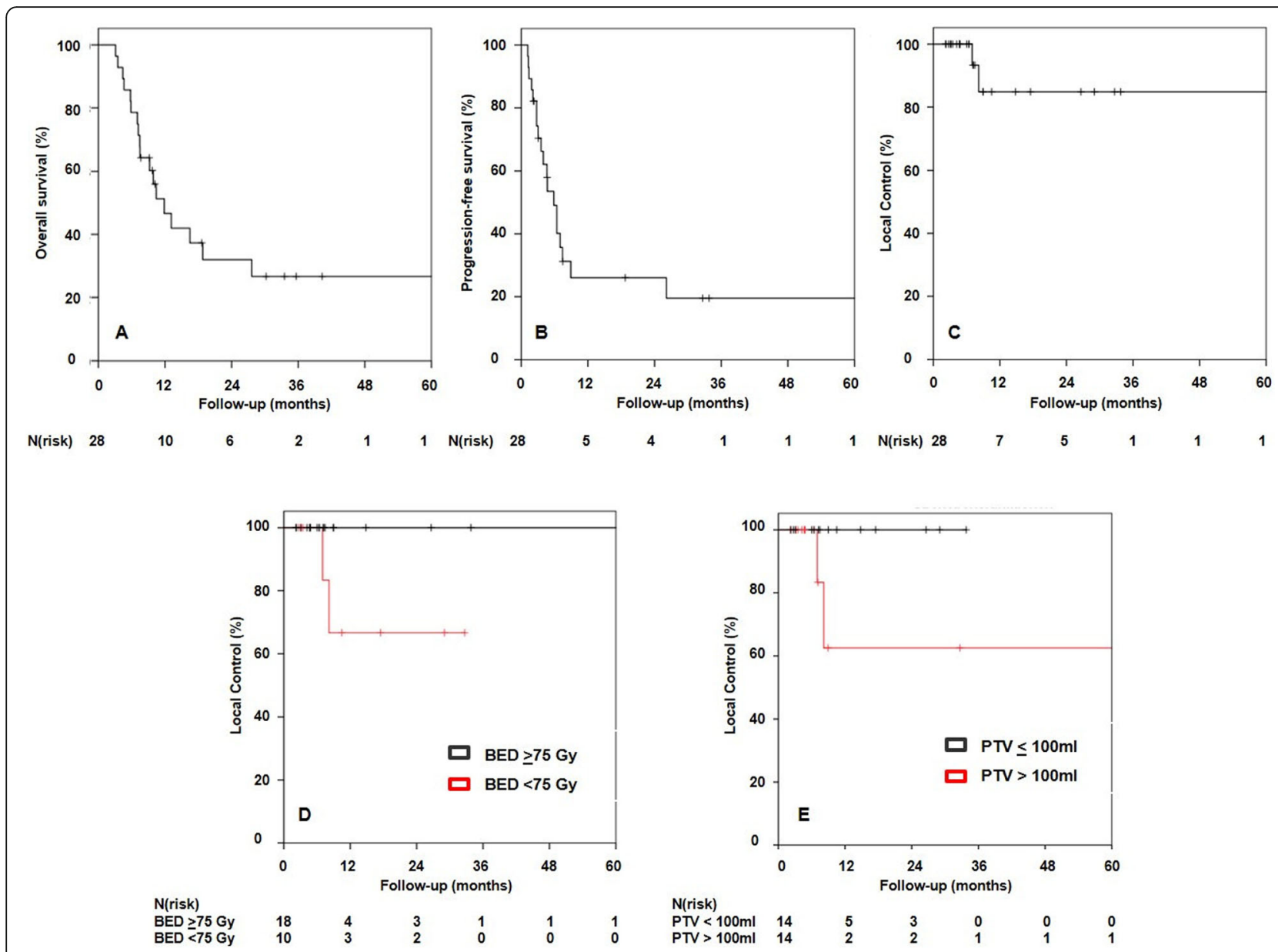

Fig. 1 Kaplan Meier survival curves for overall survival, progression-free survival and local control

As the current literature on adrenal SBRT is still limited, a patterns-of-care analysis for patients treated with hypofractionated radiotherapy to their adrenal metastases was conducted. We selected only oligometastatic or oligoprogressive patients who received high-dose SBRT to their adrenal metastases with curative intent in order to understand the role of ablative radiation in this cohort.

Table 3 Toxicity according to CTCAE criteria

\begin{tabular}{lll}
\hline & Grade $1 \mathrm{n}(\%)$ & Grade $2 \mathrm{n}(\%)$ \\
\hline Acute toxicity & $4(14.3 \%)$ & $5(17.9 \%)$ \\
Fatigue & $2(50.0 \%)$ & $4(80.0 \%)$ \\
Gastrointestinal & $2(50.0 \%)$ & $1(20.0 \%)$ \\
Abdominal pain & 0 & 0 \\
Anorexia & 0 & 0 \\
Chronic toxicity & $2(7.1 \%)$ & $1(3.2 \%)$ \\
Fatigue & $1(50.0 \%)$ & $1(100.0 \%)$ \\
Gastrointestinal & $1(50.0 \%)$ & 0 \\
Abdominal pain & 0 & 0 \\
Anorexia & 0 & 0 \\
\hline
\end{tabular}

In our study of 28 patients, adrenal SBRT led to promising local control accompanied by only mild toxicity. In detail, we detected a 1-year and 2-year LC rate of $84.8 \%$, which compare favorable to other studies [26, 27, 36-39]. A complete response was achieved in $8(29 \%)$ lesions, a partial response in $16(57 \%)$ and stability in $2(7 \%)$ of the patients according to the RECIST criteria. Two patients were diagnosed with local relapse at 7.0 and 8.2 months. Chawla et al. analyzed adrenal SBRT in 30 patients and reported 1-year and 2-year LC rates of 55 and $27 \%$, respectively [27]. Another recently published study included 33 patients and demonstrated a 1year LC of $58.1 \%$ and 2-year LC of $50 \%$ for the patients who were still alive [37]. Both Scouarnec et al. and Toesca et al. demonstrated slightly superior local control rates of 92.496.5\% after 1 year and $80.8-92.6 \%$ after 2 years [23, 40]. However, their irradiated tumors showed a median tumor volume of 13.1 and $19 \mathrm{ml}$, while the median tumor volume in our study was considerably higher with $42 \mathrm{ml}$ for the GTV and $96 \mathrm{ml}$ for the PTV. The larger tumor volumes may have comprised local control in our study. Indeed, Zhao et al. reported that a larger GTV volume significantly correlated with inferior LC following adrenal SBRT [41]. 
Similar to pulmonary and hepatic SBRT, a clear doseresponse relationship is also expected for the treatment of adrenal gland metastases $[17,42]$. Chance et al. recently reported no local failures following adrenal SBRT with a $\mathrm{BED}_{10}>100 \mathrm{~Gy}$ [26]. In line with these results, another analysis demonstrated that adrenal SBRT with a $\mathrm{BED}_{10} \geq$ 85.5 Gy correlated with superior LC [41]. Notably, the patients in the study by Scouarnec et al. were treated with higher total doses in $\mathrm{BED}_{10}$ (median 112.5 Gy) compared to the median $\mathrm{BED}_{10}$ in our analysis $(75.0 \mathrm{~Gy}$ ), which might also have impaired our results [40]. We also detected a non-significant trend for superior $\mathrm{LC}$ if a $\mathrm{BED}_{10} \geq$ 75.0 Gy was applied $(p=0.101)$, both patients diagnosed with a local recurrence were treated with a $\mathrm{BED}_{10}<67.5$ Gy. However, due to the close proximity of the adrenal glands to the stomach, the duodenum and the small bowel and their intrinsic radiosensitivity, higher doses often could not be safely delivered without potentially increasing toxicity. In this cohort, patients were treated with the highest possible doses, but organ-specific doses constraints regularly caused dose restrictions. This fact is illustrated by the wide range of calculated conformity indices in this study (median CI of 0.5 (range 0.4.-0.99), with $50 \%$ of the patients requiring reduced target volume coverage to meet adjacent OAR dose constraints.

The adrenal glands have been reported to show extensive respiration induced motion of more than $20 \mathrm{~mm}$ in several directions [22, 43]. To account for target movement due to breathing, nearly all patients were treated with a 4-D-CTbased internal target volume (ITV) concept in our study. However, Cusumano et al. recently reported that the motion amplitude acquired during 4-D-CT does not correlate to the magnitude of drifts or the necessary margins during treatment [44]. Hence, passive motion management strategies like the 4-D-CT-based ITV concept might not always be sufficient and might potentially influence local control following adrenal SBRT.

On the contrary, active motion management strategies like gating and tracking, allow for real-time target monitoring during radiotherapy [22,45]. When applying gating or tracking, the ITV-concept is no longer needed and PTV sizes can be substantially decreased. Haidenberger et al. analyzed robotic radiosurgery using active tumor tracking at the CyberKnife for adrenal SBRT and reported much smaller PTV sizes of in median $48.6 \mathrm{~cm}^{3}$ compared to the median PTV size of $95.9 \mathrm{~cm}^{3}$ in our study, in which an ITV-based approach was applied [43]. Due to the smaller PTV sizes and hence the larger distance to surrounding radiosensitive OARs, higher doses potentially leading to superior LC could be delivered when active motion management strategies are used [40, 46]. However, as gating and tracking technologies are usually applied in combination with X-ray based image-guidance, the invasive implantation of fiducial markers in the well vascularized adrenal glands becomes necessary. Scouarnec et al. reported about severe side-effects in $10.7 \%$ of the patients following fiducial implantation in the adrenal glands including hematomata and pneumothoraces [40].

Although gating and tumor tracking with robotic radiosurgery enables real-time monitoring of target motion, daily position and movement of the surrounding OARs in the abdomen is not taken into account. Substantial displacements and volume changes for the stomach, bowel, and duodenum are known to occur intra- and interfraction during adrenal SBRT [47]. In clinical routine, the applied treatment plan is based on a snapshot of the anatomy on the planning CT scan, which necessitates highly conservative dose restrictions to adjacent tissues. Holy et al. reported two patients who developed gastric and duodenal ulcers following adrenal SBRT with a BED of $72 \mathrm{~Gy}$ [48]. Interestingly, the corresponding calculated dose-volume load to the stomach and the small intestine was below the tolerance level of these organs. The authors attribute the high toxicity to a different filling of the stomach and the small intestine, which were not detected by $\mathrm{X}$-ray-based image-guidance. In our patient cohort, we performed daily image-guidance with MV- or $\mathrm{kV}-\mathrm{CT}$ and postponed the treatment if different fillings of hollow organs were detected. This might be also a reason why adrenal SBRT in our cohort was tolerated well with no acute or late $\geq$ grade III toxicity.

In contrast, MR-guided radiotherapy, which has recently become clinically available, offers superior soft-tissue contrast compared to X-ray-based techniques, gated dose delivery as well as real-time plan adaptation $[49,50]$. Daily interfractional changes in anatomy can immediately be visualized, and the treatment plan can be adjusted accordingly. Palacios et al. reported about respiration-gated, MR-guided SBRT of adrenal metastases in 17 patients, and underlined the high potential of real-time reoptimization of treatment plans to significantly improve target coverage and sparing of OARs [47]. Furthermore, a phase 1 trial of real-time adaptive MRguided radiation therapy in the treatment of oligometastatic malignancies of the abdomen enrolled 2 patients with adrenal metastases and concluded that real-time adaptive MRguided radiotherapy enabled safer delivery of SBRT and allowed doses escalation and/or simultaneous OAR sparing when the anatomy-of-the-day was favorable [51]. Based on the results of this study, we recently implemented respiration-gated MR-guided SBRT of adrenal metastases in our department, leading to the safe application of substantially higher total doses with the aim to further increase local control combined with even lower toxicity.

Limitations to this study were mainly caused by the retrospective nature of this analysis. Patient numbers were rather low, but similar to other studies, as adrenal SBRT is still not offered to many patients [3, 21, 22, 24, 36, 43, 45]. Furthermore, for increasing strength and homogeneity of 
our study, we only focused our analysis on truly oligometastatic or oligoprogressive patients and excluded all patients who were treated with palliative intent or dose. Median post-treatment follow- up time of 9.8 months makes it challenging to assess long-term LC, PFS, OS, and potentially late toxicity. This was a result of 18 patients (64.3\%) having died during follow-up, which highly reduced the analyzed timespan. Nevertheless, most of the patients died due to distant progression which emphasizes that patient selection for adrenal SBRT as well as administration of systemic therapy, both are crucial.

SBRT for adrenal metastases resulted in promising local control with only mild toxicity. Based on our study and our data, a dose-response relationship also seems to exist for adrenal SBRTs. However, we could also show that the application of sufficiently high doses is often limited by the close proximity of OARs and further dose escalations strategies and techniques (e.g. gating, tracking, adaptive radiotherapy) should be investigated in future.

\section{Abbreviations \\ BED: Biological effective dose; Cl: Conformity index; CR: Complete response; CT: Computed tomography; CTV: Clinical target volume; FDG-PET: Fluoro- deoxy-glucose positron emission tomography; FFF: Flattening filter free; GTV: Gross tumor volume; HI: Homogeneity index; IMRT: Intensity-modulated radiotherapy; ITV: Internal target volume; LC: Local control; MRI: Magnetic resonance imaging; OARs: Organs at risk; OS: Overall survival; PD: Progressive disease; PFS: Progression free survival; PR: Partial response; PTV: Planning target volume; SBRT: Stereotactic body radiotherapy; SD: Stable disease; VMAT: Volumetric modulated arc therapy}

\section{Acknowledgements}

We acknowledge financial support by Deutsche Forschungsgemeinschaft within the funding program Open Access Publishing, by the BadenWürttemberg Ministry of Science, Research and the Arts and by RuprechtKarls-Universität Heidelberg.

\section{Authors' contributions}

LK and JHR performed data collection. LK, JHR and MSS were responsible for writing and original draft preparation. LK, JHR, MFH, SK, SAK, ETM, BD, BN, FW, PH and SK performed patient treatment and clinical assessments and were responsible for radiooncological follow up documentation. LK and JH-R performed the statistical analysis. LK, JD and JHR conceived of the study and participated in its design and coordination. All the authors were responsible for data interpretation, participated in manuscript revisions, and approved the final manuscript.

\section{Funding}

This work was supported by a Heidelberg University young investigator grant to LK.

\section{Availability of data and materials}

The data used in this analysis is from publications available in the public domain.

\section{Ethics approval and consent to participate}

The Heidelberg Ethics Committee approved this study (S-627/2019).

\section{Consent for publication}

Not applicable.

\section{Competing interests}

The authors declare that they have no competing interests.

\section{Author details}

Department of Radiation Oncology, Heidelberg University Hospital, Im Neuenheimer Feld 400, 69120 Heidelberg, Germany. ${ }^{2}$ Heidelberg Institute of Radiation Oncology (HIRO), Heidelberg, Germany. ${ }^{3}$ National Center for Tumor diseases (NCT), Heidelberg, Germany. ${ }^{4}$ Clinical Cooperation Unit Radiation Oncology, German Cancer Research Center (DKFZ), Heidelberg, Germany. ${ }^{5}$ Heidelberg lon-Beam Therapy Center (HIT), Department of Radiation Oncology, Heidelberg University Hospital, Heidelberg, Germany. ${ }^{6}$ German Cancer Consortium (DKTK), partner site Heidelberg, Heidelberg, Germany

${ }^{7}$ Department of Radiation Oncology, University of California - San Francisco, San Francisco, USA.

Received: 22 October 2019 Accepted: 28 January 2020

Published online: 04 February 2020

References

1. Glomset DA. The incidence of metastasis of malignant tumors to the adrenals. Am J Cancer. 1938;32(1):57-61. https://doi.org/10.1158/aj.1938.57.

2. Lam K-Y, Lo C-Y. Metastatic tumours of the adrenal glands: a 30-year experience in a teaching hospital. Clin Endocrinol. 2002;56(1):95-101. https://doi.org/10.1046/j.0300-0664.2001.01435.x.

3. Plichta K, Camden N, Furqan M, et al. SBRT to adrenal metastases provides high local control with minimal toxicity. Adv Radiat Oncol. 2017;2(4):581-7. https://doi.org/10.1016/j.adro.2017.07.011.

4. Ippolito E, D'Angelillo RM, Fiore M, et al. SBRT: A viable option for treating adrenal gland metastases. Rep Pract Oncol Radiother. 2015;20(6):484-90. https://doi.org/10.1016/j.rpor.2015.05.009 PubMed PMID: 26696789; PubMed Central PMCID: PMCPMC4661344.

5. Soejima T, Hirota S, Hishikawa Y, et al. Radiation therapy for adrenal metastases. Nihon Igaku Hoshasen Gakkai Zasshi. 1997;57(12):801-4 PubMed PMID: 9394569.

6. Soffen $\mathrm{EM}$, Solin $\sqcup$, Rubenstein $\mathrm{JH}$, et al. Palliative radiotherapy for symptomatic adrenal metastases. Cancer. 1990;65(6):1318-20. https://doi. org/10.1002/1097-0142(19900315)65:6<1318::aid-cncr2820650611>3.0.co;2-h PubMed PMID: 2155051

7. Hellman S, Weichselbaum RR. Oligometastases. J Clin Oncol. 1995;13(1):8-10 PubMed PMID: 7799047.

8. Cheung P. Stereotactic body radiotherapy for oligoprogressive cancer. Br J Radiol. 2016;89(1066):20160251. https://doi.org/10.1259/bjr.20160251 PubMed PMID: 27556349; eng.

9. Kim C, Hoang CD, Kesarwala AH, et al. Role of Local Ablative Therapy in Patients with Oligometastatic and Oligoprogressive Non-Small Cell Lung Cancer. J Thorac Oncol. 2017;12(2):179-93. https://doi.org/10.1016/j.jtho.2016.10.012.

10. Gomez DR, Tang C, Zhang J, et al. Local Consolidative Therapy Vs, Maintenance Therapy or Observation for Patients With Oligometastatic Non-Small-Cell Lung Cancer: Long-Term Results of a Multi-Institutional, Phase II, Randomized Study. J Clin Oncol. 2019;37(18):1558-65. https://doi. org/10.1200/JCO.19.00201.

11. Palma DA, Olson R, Harrow $S$, et al. Stereotactic ablative radiotherapy versus standard of care palliative treatment in patients with oligometastatic cancers (SABR-COMET): a randomised, phase 2, open-label trial. Lancet. 2019;393(10185):2051-8. https://doi.org/10.1016/S0140-6736(18)32487-5.

12. Drake FT, Beninato T, Xiong MX, et al. Laparoscopic adrenalectomy for metastatic disease: Retrospective cohort with long-term, comprehensive follow-up. Surgery. 2019;165(5):958-64. https://doi.org/10.1016/j.surg.2018.11.008.

13. Duh QY. Laparoscopic adrenalectomy for isolated adrenal metastasis: the right thing to do and the right way to do it. Ann Surg Oncol. 2007;14(12): 3288-9. https://doi.org/10.1245/s10434-007-9569-3 PubMed PMID: 17896147: PubMed Central PMCID: PMCPMC2077915.

14. Heniford BT, Arca MJ, Walsh RM, et al. Laparoscopic adrenalectomy for cancer. Semin Surg Oncol. 1999;16(4):293-306 PubMed PMID: 10332775.

15. Zheng QY, Zhang GH, Zhang Y, et al. Adrenalectomy may increase survival of patients with adrenal metastases. Oncol Lett. 2012;3(4):917-20. https:// doi.org/10.3892/ol.2012.595 PubMed PMID: 22741018; PubMed Central PMCID: PMCPMC3362489.

16. Gunjur A, Duong C, Ball D, et al. Surgical and ablative therapies for the management of adrenal 'oligometastases' - a systematic review. Cancer Treat Rev. 2014;40(7):838-46. https://doi.org/10.1016/j.ctrv.2014.04.001 PubMed PMID: 24791623.

17. Andratschke $\mathrm{N}$, Alheid $\mathrm{H}$, Allgäuer $\mathrm{M}$, et al. The SBRT database initiative of the German Society for Radiation Oncology (DEGRO): patterns of care and 
outcome analysis of stereotactic body radiotherapy (SBRT) for liver oligometastases in 474 patients with 623 metastases [journal article]. BMC Cancer. 2018;18(1):283. https://doi.org/10.1186/s12885-018-4191-2.

18. Guckenberger M, Andratschke N, Alheit $\mathrm{H}$, et al. Definition of stereotactic body radiotherapy: principles and practice for the treatment of stage I nonsmall cell lung cancer. Strahlenther Onkol. 2014;190(1):26-33. https://doi. org/10.1007/s00066-013-0450-y PubMed PMID: 24052011; PubMed Central PMCID: PMC3889283.

19. Rieber J, Streblow J, Uhlmann L, et al. Stereotactic body radiotherapy (SBRT) for medically inoperable lung metastases-a pooled analysis of the German working group "stereotactic radiotherapy". Lung Cancer. 2016;97:51-8. https://doi.org/10.1016/j.lungcan.2016.04.012 PubMed PMID: 27237028

20. Sterzing F, Brunner TB, Ernst I, et al. Stereotactic body radiotherapy for liver tumors: principles and practical guidelines of the DEGRO working group on stereotactic radiotherapy. Strahlenther Onkol. 2014;190(10):872-81. https:// doi.org/10.1007/s00066-014-0714-1 PubMed PMID: 25091267.

21. Buergy D, Rabe L, Siebenlist K, et al. Treatment of Adrenal Metastases with Conventional or Hypofractionated Image-guided Radiation Therapy Patterns and Outcomes. Anticancer Res. 2018;38(8):4789-96. https://doi.org/ 10.21873/anticanres.12788 PubMed PMID: 30061250.

22. Katoh N, Onishi H, Uchinami Y, et al. Real-Time Tumor-Tracking Radiotherapy and General Stereotactic Body Radiotherapy for Adrenal Metastasis in Patients With Oligometastasis. Technol Cancer Res Treat. 2018; 17:1533033818809983. https://doi.org/10.1177/1533033818809983 PubMed PMID: 30407123; PubMed Central PMCID: PMCPMC6259059.

23. Toesca DAS, Koong AJ, von Eyben $R$, et al. Stereotactic body radiation therapy for adrenal gland metastases: Outcomes and toxicity. Adv Radiat Oncol. 2018;3(4):621-9. https://doi.org/10.1016/j.adro.2018.05.006 PubMed PMID: 30370363; PubMed Central PMCID: PMCPMC6200881

24. Ahmed KA, Barney BM, Macdonald OK, et al. Stereotactic body radiotherapy in the treatment of adrenal metastases. Am J Clin Oncol. 2013;36(5):509-13. https://doi.org/10.1097/COC.0b013e3182569189 PubMed PMID: 22781389.

25. Casamassima F, Livi L, Masciullo S, et al. Stereotactic radiotherapy for adrenal gland metastases: university of Florence experience. Int J Radiat Oncol Biol Phys. 2012;82(2):919-23. https://doi.org/10.1016/j.jirobp.2010.11. 060 PubMed PMID: 21300473.

26. Chance WW, Nguyen QN, Mehran R, et al. Stereotactic ablative radiotherapy for adrenal gland metastases: Factors influencing outcomes, patterns of failure, and dosimetric thresholds for toxicity. Pract Radiat Oncol. 2017;7(3):e195-203. https://doi.org/10.1016/j.prro.2016.09.005 PubMed PMID: 27743801.

27. Chawla S, Chen Y, Katz AW, et al. Stereotactic body radiotherapy for treatment of adrenal metastases. Int J Radiat Oncol Biol Phys. 2009;75(1):715. https://doi.org/10.1016/j.jirobp.2008.10.079 PubMed PMID: 19250766.

28. Hanna GG, Murray L, Patel R, et al. UK Consensus on Normal Tissue Dose Constraints for Stereotactic Radiotherapy. Clinical Oncol. 2018;30(1):5-14. https://doi.org/10.1016/j.clon.2017.09.007.

29. Park C, Papiez $L$, Zhang $S$, et al. Universal survival curve and single fraction equivalent dose: useful tools in understanding potency of ablative radiotherapy. Int J Radiat Oncol Biol Phys. 2008;70(3):847-52. https://doi.org/ 10.1016/j.jijrobp.2007.10.059 PubMed PMID: 18262098.

30. Kataria T, Sharma K, Subramani V, et al. Homogeneity Index: An objective tool for assessment of conformal radiation treatments. J Med Phys. 2012; 37(4):207-13. https://doi.org/10.4103/0971-6203.103606 PubMed PMID: 23293452; PubMed Central PMCID: PMCPMC3532749.

31. Shaw $E$, Kline $R$, Gillin $M$, et al. Radiation therapy oncology group: radiosurgery quality assurance guidelines. Int J Radiat Oncol Biol Phys. 1993;27(5):1231-9. https://doi.org/10.1016/0360-3016(93)90548-a PubMed PMID: 8262852

32. Al-Shafa F, Arifin AJ, Rodrigues GB, et al. A Review of Ongoing Trials of Stereotactic Ablative Radiotherapy for Oligometastatic Cancers: Where Will the Evidence Lead? [Mini Review]. Front Oncol. 2019 9(543). doi: https://doi. org/10.3389/fonc.2019.00543. English.

33. Kumar R, Xiu Y, Yu JQ, et al. 18F-FDG PET in Evaluation of Adrenal Lesions in Patients with Lung Cancer. J Nucl Med. 2004;45(12):2058-62.

34. Stephens SJ, Moravan MJ, Salama JK. Managing patients with Oligometastatic non-small-cell lung Cancer. J Oncol Pract. 2018;14(1):23-31. https://doi.org/10.1200/jop.2017.026500 PubMed PMID: 29324212.

35. Thomsen B, Fairchild A. Adrenal Oligometastases Secondary to Non-small Cell Lung Cancer-What is the Optimal Treatment Approach? Oncol Hematol Rev. 2017;13(117). https://doi.org/10.17925/OHR.2017.13.02.117.

36. Rudra S, Malik R, Ranck MC, et al. Stereotactic body radiation therapy for curative treatment of adrenal metastases. Technol Cancer Res Treat. 2013;
12(3):217-24. https://doi.org/10.7785/tcrt.2012.500320 PubMed PMID: 23369155.

37. Burjakow K, Fietkau R, Putz F, et al. Fractionated stereotactic radiation therapy for adrenal metastases: contributing to local tumor control with low toxicity. Strahlenther Onkol. 2019;195(3):236-45. https://doi.org/10.1007/ s00066-018-1390-3 PubMed PMID: 30374590.

38. Franzese C, Franceschini D, Cozzi L, et al. Minimally Invasive Stereotactical Radio-ablation of Adrenal Metastases as an Alternative to Surgery. Cancer Res Treat. 2017;49(1):20-8. https://doi.org/10.4143/crt.2016.057 PubMed PMID: 27121718; PubMed Central PMCID: PMCPMC5266381.

39. Scorsetti M, Alongi F, Filippi AR, et al. Long-term local control achieved after hypofractionated stereotactic body radiotherapy for adrenal gland metastases: a retrospective analysis of 34 patients. Acta Oncol. 2012;51(5):618-23. https:// doi.org/10.3109/0284186X.2011.652738 PubMed PMID: 22263925.

40. Scouarnec C, Pasquier D, Luu J, et al. Usefulness of Stereotactic Body Radiation Therapy for Treatment of Adrenal Gland Metastases. Front Oncol. 2019;9:732. https://doi.org/10.3389/fonc.2019.00732 PubMed PMID: 31448234; PubMed Central PMCID: PMCPMC6692476.

41. Zhao X, Zhu X, Fei J, et al. Short-term outcomes and clinical efficacy of stereotactic body radiation therapy (SBRT) in treatment of adrenal gland metastases from lung cancer. Radiat Oncol. 2018;13(1):205. https://doi.org/ 10.1186/s13014-018-1152-5 PubMed PMID: 30348187; PubMed Central PMCID: PMCPMC6196411.

42. Onishi $H$, Shirato $H$, Nagata $Y$, et al. Hypofractionated stereotactic radiotherapy (HypoFXSRT) for stage I non-small cell lung cancer: updated results of 257 patients in a Japanese multi-institutional study. J Thorac Oncol. 2007;2(7 Suppl 3):S94-100. https://doi.org/10.1097/JTO. 0b013e318074de34 PubMed PMID: 17603311.

43. Haidenberger A, Heidorn SC, Kremer N, et al. Robotic Radiosurgery for Adrenal Gland Metastases. Cureus. 2017;9(3):e1120. https://doi.org/10.7759/ cureus.1120 PubMed PMID: 28451479; PubMed Central PMCID: PMCPMC5406171.

44. Cusumano D, Dhont J, Boldrini $L$, et al. Predicting tumour motion during the whole radiotherapy treatment: a systematic approach for thoracic and abdominal lesions based on real time MR. Radiother Oncol. 2018;129(3):45662. https://doi.org/10.1016/j.radonc.2018.07.025 PubMed PMID: 30144955.

45. Desai A, Rai H, Haas J, et al. A Retrospective Review of CyberKnife Stereotactic Body Radiotherapy for Adrenal Tumors (Primary and Metastatic): Winthrop University Hospital Experience. Front Oncol. 2015;5: 185. https://doi.org/10.3389/fonc.2015.00185 PubMed PMID: 26347852; PubMed Central PMCID: PMCPMC4538288.

46. Li J, Shi Z, Wang Z, et al. Treating adrenal tumors in 26 patients with CyberKnife: a mono-institutional experience. PLoS One. 2013;8(11):e80654. https://doi.org/10.1371/journal.pone.0080654 PubMed PMID: 24278303; PubMed Central PMCID: PMCPMC3835566.

47. Palacios MA, Bohoudi O, Bruynzeel AME, et al. Role of daily plan adaptation in MR-guided stereotactic ablative radiation therapy for adrenal metastases. Int J Radiat Oncol Biol Phys. 2018;102(2):426-33. https://doi.org/10.1016/j. ijrobp.2018.06.002 PubMed PMID: 29902559.

48. Holy $R$, Piroth $M$, Pinkawa $M$, et al. Stereotactic body radiation therapy (SBRT) for treatment of adrenal gland metastases from non-small cell lung cancer. Strahlenther Onkol. 2011;187(4):245-51. https://doi.org/10.1007/ s00066-011-2192-z PubMed PMID: 21424513.

49. Mutic S, Dempsey JF. The ViewRay system: magnetic resonance-guided and controlled radiotherapy. Semin Radiat Oncol. 2014;24(3):196-9. https://doi. org/10.1016/j.semradonc.2014.02.008 PubMed PMID: 24931092.

50. Raaymakers BW, Jurgenliemk-Schulz IM, Bol GH, et al. First patients treated with a 1.5 T MRI-Linac: clinical proof of concept of a high-precision, highfield MRI guided radiotherapy treatment. Phys Med Biol. 2017;62(23):L41-50. https://doi.org/10.1088/1361-6560/aa9517 PubMed PMID: 29135471.

51. Henke L, Kashani R, Robinson C, et al. Phase I trial of stereotactic MR-guided online adaptive radiation therapy (SMART) for the treatment of oligometastatic or unresectable primary malignancies of the abdomen. Radiother Oncol. 2018;126(3):519-26. https://doi.org/10.1016/j.radonc.2017. 11.032 PubMed PMID: 29277446.

\section{Publisher's Note}

Springer Nature remains neutral with regard to jurisdictional claims in published maps and institutional affiliations. 